\title{
Commentary: Synaptic Excitation in Spinal Motoneurons Alternates with Synaptic Inhibition and Is Balanced by Outward Rectification during Rhythmic Motor Network Activity
}

\author{
Rune W. Berg* \\ Department of Neuroscience, University of Copenhagen, Copenhagen, Denmark
}

Keywords: spinal cord, central pattern generators (CPG), balanced networks, rhythm generation, motor control, patch-clamp, voltage-clamp

\section{A commentary on}

Synaptic Excitation in Spinal Motoneurons Alternates with Synaptic Inhibition and Is Balanced by Outward Rectification during Rhythmic Motor Network Activity

by Guzulaitis, R., and Hounsgaard, J. (2017). J. Neurosci. 37, 9239-9248. doi: 10.1523/JNEUROSCI.0800-17.2017

In a recent study, Guzulaitis and Hounsgaard (2017) (GH2017) used whole cell voltage clamp (VC) on the reversal potential for inhibition or excitation to assess their synaptic currents (Johnston and Wu, 1995; Brette and Destexhe, 2012). GH2017 concluded that inhibition and excitation alternated

OPEN ACCESS

Edited by:

David Parker,

University of Cambridge,

United Kingdom

Reviewed by:

Wen-Chang $L i$

University of St Andrews,

United Kingdom

*Correspondence:

Rune W. Berg

runeb@sund.ku.dk

Received: 25 November 2017 Accepted: 04 January 2018 Published: 18 January 2018

Citation:

Berg RW (2018) Commentary:

Synaptic Excitation in Spinal Motoneurons Alternates with Synaptic Inhibition and Is Balanced by Outward Rectification during Rhythmic Motor

Network Activity.

Front. Neural Circuits 12:1.

doi: 10.3389/fncir.2018.00001 during rhythmic scratching, and a voltage-dependent intrinsic conductance was masking this input such that it appeared as balanced excitation and inhibition in previous published work (Berg et al., 2007; Petersen et al., 2014). Nevertheless, this reasoning relies entirely on the validity of the clamp and, as we will see below, there is a clamp error, which complicates the interpretation of their data. Errors associated with voltage-clamp is a common problem as noted in previous reports (Spruston et al., 1993; Williams and Mitchell, 2008; Petersen, 2017).

The membrane current (I) is composed of intrinsic, leak, excitatory and inhibitory currents with individual conductances and reversal potentials, which collectively form a membrane resistance $\left(R_{m}\right)$ and an equilibrium potential $\left(E_{m}\right)$. When recording these using a pipette electrode, its resistance $\left(R_{s}\right)$, sometimes called access or series resistance, is in series with $R_{m}$ (Figure 1A). When there is no electrode current the membrane potential $V_{m}=E_{m}$. However, during VC, a non-zero current introduces a drop in potential over $R_{s}$, which can only be partially compensated with the amplifier electronics (Brette and Destexhe, 2012). $R_{s}$ therefore has an uncompensated part (blue, $R_{u s}$, Figures 1A,B), which generates an unaccounted drop in potential from the clamp potential $\left(V_{c}\right)$ proportional to the pipette current:

$$
V_{m}=V_{c}-I \cdot R_{u s}
$$

GH2017 report: "Voltage clamp (VC) experiments were performed on motoneurons when access resistance was low $\left(R_{a}<20 \mathrm{M} \Omega\right)$ and possible to compensate by $60-80 \%$." This means that $R_{u s}=$ $20-40 \% \cdot 20 \mathrm{M} \Omega=4-8 \mathrm{M} \Omega$. When clamping at $0 \mathrm{mV}$ the applied current is likely large. The authors do not report $I$ for their clamp experiments (Figures 8-9), but their IV-plots suggest up to $10 \mathrm{nA}$ (Figures 5E, 6). Hence, when trying to clamp at $0 \mathrm{mV}, V_{m}$ is really $-10 \mathrm{nA} \cdot 4 \mathrm{M} \Omega=-40 \mathrm{mV}$ with $80 \% R_{\mathcal{S}}$-compensation. 

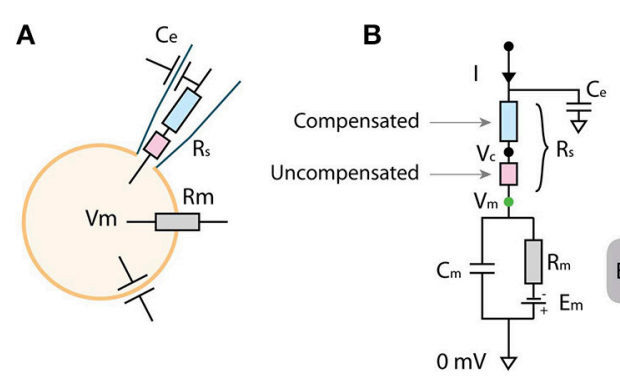

$\mathrm{Em}_{\mathrm{m}}=\frac{\mathrm{gi}^{*} \mathrm{Ei}+\mathrm{ge} \mathrm{e}^{*} \mathrm{Ee}+\sum \mathrm{gj}{ }^{*} \mathrm{Ej}}{\mathrm{g} \text { total }}$

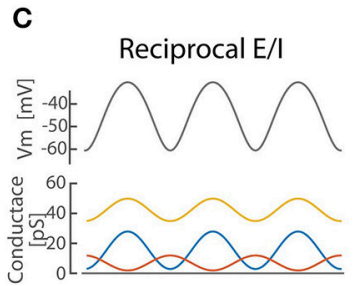

D
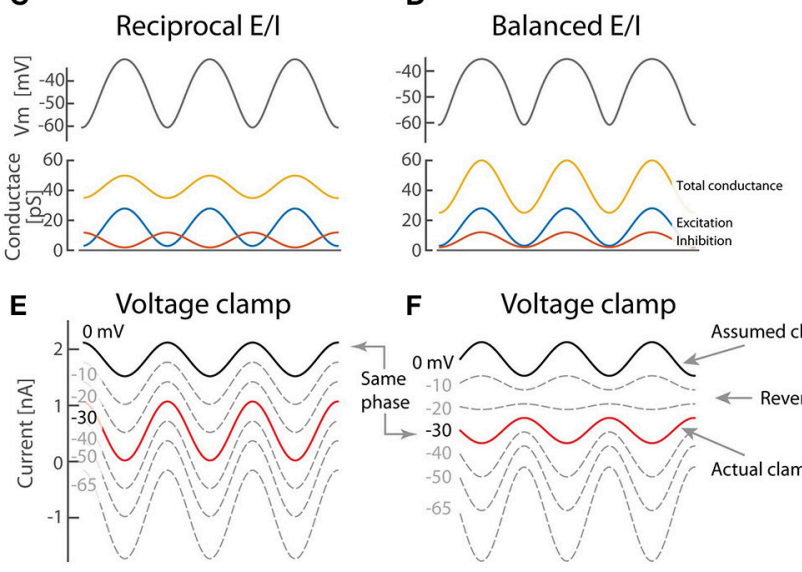

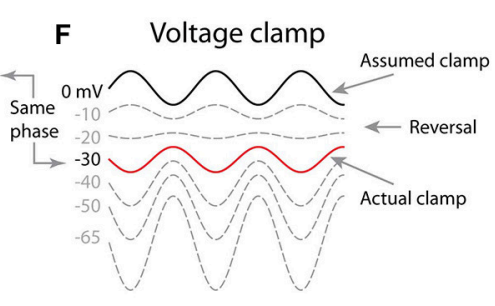

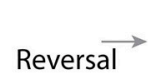
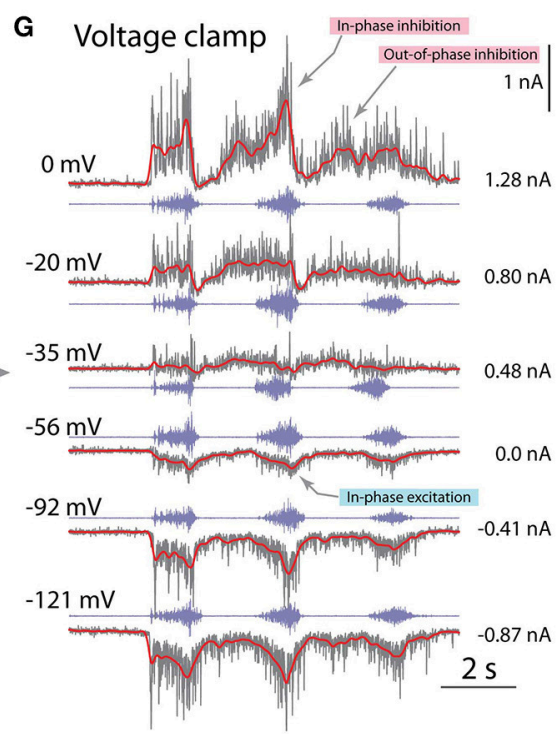

H Control

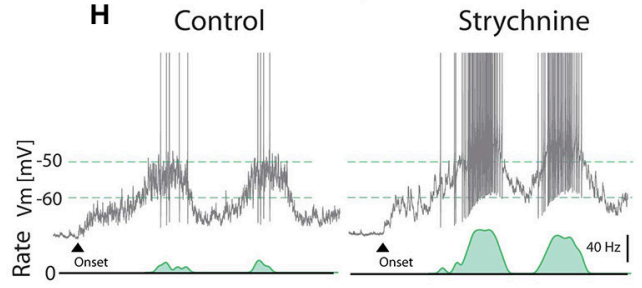

FIGURE 1 | Caveats using voltage clamp to resolve excitation and inhibition. (A) Whole-cell VC can be decomposed into electrical components including the pipette series resistance $\left(R_{S}\right)$. (B) Partial compensation for $R_{S}$ introduces a disparity between clamped potential $\left(V_{C}\right)$ and $V_{m}$ due to uncompensated resistance (red). (C) Reciprocal model for rhythmic $V_{m}$ has alternating E/I. $\left(R_{m}=20 \mathrm{M} \Omega\right.$, $\left.E_{\text {leak }}=-70 \mathrm{mV}\right)$. (D) Balanced model has concurrent E/I and also rhythmic $V_{m}$. (E) Outward currents measured using VC is assumed to be inhibition when clamping OmV (black). The actual clamp is at $-30 \mathrm{mV}$ (red). (F) Balanced E/I spuriously appears as reciprocal when the actual clamp is below synaptic reversal potential ("same phase" cf. red in $\mathbf{F}$ and black in E). (G) VC-recording of a putative motoneuron with blocked spikes (with intracellular QX314) at different holding potentials (gray: current, red: mean, blue: nerve). Reversal of phase (arrow) is consistent with the balanced scheme (F) although with a smaller out-of-phase inhibition (indicated). (H) Blocking inhibition (strychnine) increases firing rate also consistent with the balanced scheme. (G) provided by A. Alaburda (current levels indicated, right) and (H) adapted with permission (Vestergaard and Berg, 2015).

To better understand the issue, we consider steady-state where all current passes through the resistors. From Ohm's law the voltage drop over $R_{u s}$ is $V_{m}-V_{c}=I \cdot R_{u s}$. Similarly, the voltage drop over the membrane is $E_{m}-V_{m}=I \cdot R_{m}$. Combining these we can eliminate $I$ and isolate $V_{m}$ :

$$
V_{m}=\frac{V_{c} R_{m}+E_{m} R_{u s}}{R_{m}+R_{u s}}
$$

Hence, for a good clamp $\left(V_{m} \approx V_{c}\right)$ it is required that $R_{m} \gg R_{u s}$. $\mathrm{GH} 2017$ report a membrane conductance of $49.2 \mathrm{nS}$ (Figure $5 \mathrm{~B}$ ), which gives $R_{m}=20 M \Omega$. With these values $\left(E_{m}=-70 m \mathrm{~V}\right)$ clamping at $0 \mathrm{mV}$ gives

$$
V_{m}=\frac{0-70 m V \cdot 8 M \Omega}{28 M \Omega}=-20 m V
$$

Whereas $R_{u s}$ is assumed constant, $R_{m}$ may change dramatically due to synaptic and intrinsic conductance. GH2017 nicely document a nonlinearity starting at $-30 \mathrm{mV}$ (Figures 5,6 ), and a conductance of $314 \mathrm{nS}\left(R_{m}=3.2 \mathrm{M} \Omega\right)$. Here, the low $R_{m}$ even becomes smaller than $R_{u s}$ and therefore the clamp deteriorates further:

$$
V_{m}=\frac{0-70 m V \cdot 8 M \Omega}{11.2 M \Omega}=-50 m V
$$

The clamp is unlikely to be this bad, since the reduction in $R_{m}$ occurs above $-50 \mathrm{mV}$. Also, $E_{m}$, which we assume constant, may depolarize due to change in the weighted average (Figure 1B), which mitigates the effect. The exact level of clamping of $V_{m}$ with $\left(V_{c}=0 \mathrm{mV}\right)$ is difficult to estimate and may change in time. A reasonable guess is around $V_{m}=-30 \mathrm{mV}$.

What is the consequence of this clamping error? To address this question, we use a one-compartment model receiving either reciprocal (Figure 1C) or concurrent (balanced) excitation and inhibition (E/I) (Figure 1D), which are the schemes that GH2017 intended to distinguish between. Both result in rhythmic $V_{m}$, although the effect of balanced E/I may seem counter-intuitive (Kolind et al., 2012; Petersen et al., 2014). The problem appears when presuming the outward current is inhibition, when setting $V_{c}=0 \mathrm{mV}$ (assumed clamp, black line Figures 1E,F). From 
the above, we know that the actual clamp is likely at $-30 \mathrm{mV}$ (red traces). Here, the phase of the outward current reverses making the actual clamp in the balanced scheme (red, F) appear qualitatively similar to the assumed clamp in the reciprocal (black, E). Therefore, the VC experiments by GH2017 are difficult to interpret and ill-suited to discriminate between these schemes.

Although reciprocal E/I is a widely held belief in the literature, there is remarkably little experimental support in tetrapod vertebrates. The Ia-inhibitory interneuron has reciprocal activity (Geertsen et al., 2011), whereas the Renshaw interneuron has recurrent inhibition, both connected to motoneurons. Nevertheless the action of the remaining inhibitory population is largely unexplored. The scarcity in experimental reports that resolve E/I input is likely due to nonlinear properties and difficulties in separating synaptic current, although methods have been proposed (Berg and Ditlevsen, 2013; Vich et al., 2017). Space clamp issues also confounds the separation of E/I (Chadderton et al., 2014). Previous observations in turtles based on current-clamp indicated concurrent E/I. Here, voltageactivated conductances were circumvented by injecting negative current to hyperpolarize $V_{m}$ below the onset of the IVnonlinearity. Therefore the disparity between reports cannot be attributed to outward rectification, as otherwise suggested by GH2017, see e.g., Figure 3A in Berg et al. (2007) and Figures 2-4 in Berg et al. (2008). Further, VC experiments were performed using sharp electrodes where spikes were blocked by pharmacology (QX314). QX314 likely also has the advantage of increasing $R_{m}$, thus improving the $R_{m} \gg$ $R_{u s}$ requirement (Monier et al., 2008). A current-reversal was observed in accordance with the balanced scheme (Figure 1G). Other experiments confirm that when blocking excitation and inhibition pharmacologically, the high conductance vanish even

\section{REFERENCES}

Berg, R. W. (2017). Neuronal population activity in spinal motor circuits: greater than the sum of its parts. Front. Neural Circ. 11:103. doi: 10.3389/fncir.2017.00103

Berg, R. W., Alaburda, A., and Hounsgaard, J. (2007). Balanced inhibition and excitation drive spike activity in spinal half-centers. Science 315, 390-393. doi: $10.1126 /$ science. 1134960

Berg, R. W., and Ditlevsen, S. (2013). Synaptic inhibition and excitation estimated via the time constant of membrane potential fluctuations. J. Neurophysiol. 110, 1021-1034. doi: 10.1152/jn.00006.2013

Berg, R. W., Ditlevsen, S., and Hounsgaard, J. (2008). Intense synaptic activity enhances temporal resolution in spinal motoneurons. PLOS ONE 3:e3218. doi: 10.1371/journal.pone.0003218

Brette, R., and Destexhe, A. (eds.). (2012). "Intracellular recording," in Handbook of Neural Activity Measurement (New York, NY: Cambridge University Press), 44-91.

Chadderton, P., Schaefer, A. T., Williams, S. R., and Margrie, T. W. (2014). Sensory-evoked synaptic integration in cerebellar and cerebral cortical neurons. Nat. Rev. Neurosci. 15, 71-83. doi: 10.1038/nrn3648

Geertsen, S. S., Stecina, K., Meehan, C. F., Nielsen, J. B., and Hultborn, H. (2011). Reciprocal Ia inhibition contributes to motoneuronal hyperpolarisation during the inactive phase of locomotion and scratching in the cat. J. Physiol. 589, 119-134. doi: 10.1113/jphysiol.2010.199125

Guzulaitis, R., and Hounsgaard, J. (2017). Synaptic excitation in spinal motoneurons alternates with synaptic inhibition and is balanced by outward at the same $V_{m}$, suggesting that conductance increase is caused by synaptic input rather than voltage-activated conductances (Figure 8 in Berg and Ditlevsen, 2013). Application of strychnine had a strong depolarizing effect (Figure 1H) especially in the onphase, which is also difficult to reconcile with the reciprocal E/I scheme (Berg et al., 2007; Vestergaard and Berg, 2015).

Contrary to the conclusions of GH2017, these observations suggest that a substantial fraction of the spinal neurons receive concurrent E/I, which may not exclude that others receive reciprocal. In fact, the neuronal population is divided between irregular and regular spiking, suggesting some receive reciprocal and others receive balanced input most likely on a spectrum between the two (Petersen and Berg, 2016; Berg, 2017). Notice in addition to the in-phase $\mathrm{E} / \mathrm{I}$ there is also a weaker out-ofphase inhibition (Figure 1G). Spinal motor pattern generation may therefore be more complex and not exclusively conform to either of the schemes (Kishore et al., 2014).

\section{AUTHOR CONTRIBUTIONS}

The author confirms being the sole contributor of this work and approved it for publication.

\section{FUNDING}

Funded by Danish Council for Independent Research Medical Sciences and the Dynamical Systems Interdisciplinary Network, University of Copenhagen.

\section{ACKNOWLEDGMENTS}

Thanks to A. Alaburda for providing data presented in Figure 1G.

rectification during rhythmic motor network activity. J. Neurosci. 37, 92399248. doi: 10.1523/JNEUROSCI.0800-17.2017

Johnston, D., and Wu, S.-S. (1995). Foundations of Cellular Neurophysiology, Cambridge, MA: MIT Press.

Kishore, S., Bagnall, M. W., and McLean, D. L. (2014). Systematic shifts in the balance of excitation and inhibition coordinate the activity of axial motor pools at different speeds of locomotion. J. Neurosci. 34, 14046-14054. doi: 10.1523/JNEUROSCI.0514-14.2014

Kolind, J., Hounsgaard, J., and Berg, R. W. (2012). Opposing effects of intrinsic conductance and correlated synaptic input on Vm-fluctuations during network activity. Front. Comput. Neurosci. 6:40. doi: 10.3389/fncom.2012.00040

Monier, C., Fournier, J., and Frégnac, Y. (2008). In vitro and in vivo measures of evoked excitatory and inhibitory conductance dynamics in sensory cortices. J. Neurosci. Methods 169, 323-365. doi: 10.1016/j.jneumeth.2007.11.008

Petersen, C. C. (2017). Whole-cell recording of neuronal membrane potential during behavior. Neuron 95, 1266-1281. doi: 10.1016/j.neuron.2017.06.049

Petersen, P. C., and Berg, R. W. (2016). Lognormal firing rate distribution reveals prominent fluctuation-driven regime in spinal motor networks. eLife 5:e18805. doi: 10.7554/eLife.18805

Petersen, P. C., Vestergaard, M., Jensen, K. H., and Berg, R. W. (2014). Premotor spinal network with balanced excitation and inhibition during motor patterns has high resilience to structural division. J. Neurosci. 34, 2774-2784. doi: 10.1523/JNEUROSCI.3349-13.2014

Spruston, N., Jaffe, D. B., Williams, S. H., and Johnston, D. (1993). Voltage- and space-clamp errors associated with the measurement of electrotonically remote synaptic events. J. Neurophysiol. 70, 781-802. 
Vestergaard, M., and Berg, R. W. (2015). Divisive gain modulation of motoneurons by inhibition optimizes muscular control. J. Neurosci. 35, 3711-3723. doi: 10.1523/JNEUROSCI.3899-14.2015

Vich, C., Berg, R. W., Guillamon, A., and Ditlevsen, S. (2017). Estimation of synaptic conductances in presence of nonlinear effects caused by subthreshold ionic currents. Front. Comput. Neurosci. 11:69. doi: 10.3389/fncom.2017. 00069

Williams, S. R., and Mitchell, S. J. (2008). Direct measurement of somatic voltage clamp errors in central neurons. Nat. Neurosci. 11, 790-798. doi: $10.1038 / \mathrm{nn} .2137$
Conflict of Interest Statement: The author declares that the research was conducted in the absence of any commercial or financial relationships that could be construed as a potential conflict of interest.

Copyright (c) 2018 Berg. This is an open-access article distributed under the terms of the Creative Commons Attribution License (CC BY). The use, distribution or reproduction in other forums is permitted, provided the original author(s) or licensor are credited and that the original publication in this journal is cited, in accordance with accepted academic practice. No use, distribution or reproduction is permitted which does not comply with these terms. 